\title{
A Reliability Simulation of Warm-fund and Redundancy Repairable System
}

\author{
Shouzhu Wang \\ Department of Science, Yanshan University(west campus) \\ PO box 1447, Qinhuangdao 066004, China \\ E-mail: wangshouzhu08@126.com \\ Xianyun Meng (Corresponding author) \\ Department of Science, Yanshan University(west campus) \\ Qinhuangdao 066004, China \\ Yuanyuan Li \\ Department of Science, Yanshan University \\ Qinhuangdao 066004, China \\ Tianchong Zhou \\ Department of Science, Yanshan University \\ Qinhuangdao 066004, China
}

Supported by the Plan Projects of He Bei Education Office (No.2007323)

Supported by the Foundation for the natural science of He Bei province of China (A2005000301)

\begin{abstract}
In this paper, we give analysis to the reliabilities and simulations of the warm-fund and redundancy maintenance system. First, we analyze the relationship between the status of components and the system's minimal cut-set. And, we simulate the system by Monte Carlo, get the detailed diagram, program of simulation, and calculation of indicators. The last, we give one example to shows the feasibility of this method.
\end{abstract}

Keywords: Fault tree, Repairable system, Warm-fund

\section{Introduction}

For long time, it has been a hard problem which is how to evaluate reliability of a complicated and repairable system in quantificational, the classical treatment is to utilize Markov process to describe system's state, consequently compute various dependability parameters of system. But only a few special types of systems that can be computed by the classical treatment, and then it seem to be incapable to face numerous complicatedly repairable systems. Moreover, along with the science technical progress, the system is more and more complication and multi-function, if it has no higher credibility, then will go wrong continually after the system circulate, and system will be serious influenced, so the request to the reliability of system is higher, but the system design difficulty will be increased and the expenses will be increased. Therefore, urgently need a kind of kill two birds with one stone a method. Along with the computer science soon develop in recent years, simulation technique has already got an extensive application with the base of Monte Carlo method in many engineering realms, and people start try to use to simulation technique to solve this hard problem, and it has already become main means to evaluate the reliability of complicatedly repairable systems in quantitative evaluate. And many domestic and international papers all have some cases which analyses the reliability in simulation approach, for example the paper $(\mathrm{Xu}, 2007)$ apply simulation approach to research the system considering components stand-by redundancy.

This paper considers warm-fund and redundancy repairable system. And put fault tree analysis and simulation approach 
to combine together, according to minimal cut-set to judge the state of system, then in Monte Carlo method to imitate, finally we can get the reliability index. The result indicates the simulation model is viable in case, and that offer viable method for analyzing and improving the system reliability design.

\section{System model}

\subsection{System descriptions and assumption}

The system is constituted by $n$ parts and a repairing equipment, each part has $y_{i}$ warm-fund and redundancy, system or parts only have two states: failure and good; When the system is running, if part is failure and the number of redundancy amount $y_{i}>0$, then the failure part is replaced by the new one, we take no account of time to lose, repairing equipment maintains immediately the bad one; When the repairing equipment works, if have part lapse at this time, then the part need to wait for maintaining; While making better a part, if this kind of part has a part in the work, this part will be storage and redundancy, and otherwise this part immediately gets into work.

Further assumption: Record $x_{i}$ means the part state, the $x_{i}=1$ means the part normally works and $x_{i}=0$ means the part break down; The work life, storage life and repairing time to all obey index distributing, respective parameter: $\lambda_{i}, \lambda_{2}, \mu$; Each one random variable is mutual independence; all parts can be repaired to new; each part is all new when system starts circulate.

\subsection{System appearances judge}

We simulate system's de running state by simulating running state of system's parts, then we get system's imitating model, though the model is substantially and all ever-changing, is the causal tool of reflecting the basic event lapses and system lapses, and system's fault tree reflects this kind of relation. At course of analyzing fault tree, have a very important concept: namely minimal cut-set of fault tree. In the fault tree, the gather of basic affairs of causing tip affair occurring is called to cut-set, if arbitrarily throw away a basic affair in cut-set and then don't be cut-set, so such of cut-set be minimal cut-set. In fault tree minimal cut-set generally not only one. Can know according to the above definition, as long as have one cut-set occur, the tip affair takes place in fault tree and the system lose efficacy.

System's minimal cut-set matrix is $C=\left[c_{j i}\right]_{m \times n}$, namely the system has $m$ minimal cut-set, and $c_{j i}=0$ mean the $i$ part includes in cut-set $j$ and otherwise record $c_{j i}=1$. When the system circulates, the parts in the work appear breakdown, if corresponding redundancy parts $y_{i}>0$ at this time, immediately switch, otherwise need to judge that whether parts' fault will cause system's fault, mark $x_{i}=0$, at this time the parts' vector of state is $X=\left\{x_{1}, x_{2}, \cdots, x_{n}\right\}$. Then compare vector $X$ and each row of minimal cut-set matrix $C$, if exist certain $j(j=1,2, \cdots, m)$, make for all $i(i=1,2, \cdots, n)$ and all have $c_{j i} \geq x_{i}$, so the system breaks down.

\section{Computing index of reliability}

The number of simulative times is $\mathrm{N}$, system's furthest working hours is $T_{\text {max }}$, put it divide into $m$ equal small zone, distant time is $\Delta T$. Counting the number of times about system lapse in the zone $\left(t_{i-1}, t_{i}\right]$, use $m_{i}$ to figure.

\subsection{The not credibility of system $F(t)$}

$F(t)=\frac{\left(\sum_{i=1}^{k} m_{i}\right)}{N}$, among them $k=\left\lceil\frac{t}{\Delta t}\right\rceil+1$.

3.2 The credibility of system $R(t)$

$R(t)=1-F(t)=1-\frac{\sum_{i=1}^{i=k} m_{i}}{N}$, among them $k=\left\lceil\frac{t}{\Delta t}\right\rceil+1$.

3.3 The system's lapse density function $f(t)$

$f(t)=\frac{m_{i}}{\Delta T \cdot N}$, among them $i=\left\lceil\frac{t}{\Delta t}\right\rceil+1$.

3.4 The system lapse efficiency $\lambda(t)$

$\lambda(t)=\frac{f(t)}{R(t)}=\frac{m_{i}}{\Delta T\left(N-\sum_{i=1}^{k}\right) m_{i}}$ among them, $i=\left\lceil\frac{t}{\Delta t}\right\rceil+1$.

3.5 System average life $M T B F$

$M T B F=\frac{1}{N} \sum_{i=1}^{N} T_{i}$

3.6 Parts probability importance $W_{N}\left(Z_{i}\right)$

$$
W_{N}\left(Z_{i}\right)=\frac{\text { the times which parts lapse causes system lapse }}{\text { the all times of system lapse }}
$$




\subsection{The parts structure importance $W\left(Z_{i}\right)$}

$$
W\left(Z_{i}\right)=\frac{\text { the times which parts lapse cause system lapse }}{\text { the all times of parts lapse }}
$$

Axioms: In all parts in the system, improve maximal credibility of part that the probability importance is biggest, will make the system credibility get the biggest improvement.

Prove: When the parts credibility change $\Delta p_{j} j=1, \cdots, n$, base the definition of parts probability importance (Cao, 1986), the alterative quantity of system credibility is:

$$
\Delta h=\sum_{j=1}^{n} \frac{\partial h(p)}{\partial p_{j}} \Delta p_{j}=\sum_{j=1}^{n} I_{h}(j) \Delta p
$$

Therefore, when the credibility of part $j$ increases $\Delta p$, system credibility increases $\Delta h_{j}=I_{h}(j) \Delta p$. Among them $h(p)$ is system credibility, $p_{j}$ is part credibility, $I_{h}(j)$ is part probability importance. So, if probability importance of the part $k$ is the biggest and then improve credibility of part $k$ will make the credibility of system get the biggest improve in all parts.

\section{Simulative model and arithmetic}

\subsection{Simulative logic flow chart}

The system's Simulative logic flow chart is shown as figure 1.

Carry on initializing towards simulative data first while carrying on simulating each time, get some time according to distributing function of the parts failure and the repair time, choose to take place at the earliest time, shall simulate clock to push forward; Continue to judge above-mentioned at the earliest time from what affairs cause of, renew different variable according to the different affairs, if part within work breaks down, then judge whether have redundancy, if have the redundancy parts immediately switch, simulation continues to carrying on, otherwise judge whether this fault causes system fault or not, to decide to whether this simulative be over or continue to circulate.

\subsection{Simulative process}

\subsubsection{The setting and reading of basic data}

The basic data includes: simulative times of system $N$, simulative times $r=0$ at present, the number of system's parts $n$, redundancy amount of each part $y_{i}$, parameter of parts' life and repairing time, the number of minimal cut-set and minimal cut-set matrix $C$ by analyzing fault tree to get.

4.2.2 Judge whether simulating really be over pass to simulative times.

If simulative times at present $r>N$, simulation be over and turn to step 4.2.6; Otherwise simulating continues to carrying on.

\subsubsection{Variable initialization}

Carry on initializing towards variable first before carrying on simulating each time, includes parts state vector $X=$ $\{1,1, \cdots, 1\}$, redundancy amount $y=\left\{y_{1}, y_{2}, \cdots, y_{n}\right\}$, the numbers of fault parts $k=0$, this simulating keep on time $T_{r}=0$.

4.2.4 According to the parameters of parts work and storage life and give birth to lapse time for each normal part: $T_{f 1}, T_{f 2}, \cdots, T_{f n}, T_{z 1}, T_{z 2}, \cdots, T_{z n}$; According to the parameters of parts' repair life and give birth to repairing time for each repairing part: $T_{a}$. Choose to take place at the earliest time in all time, if take for $T_{x}$, will push simulative clock, $T_{r}=T_{r}+T_{x}$

\subsubsection{Judging $T_{x}$ adscription}

Judging whose the time $T_{x}$ is: a work part fault or a redundancy part fault, or a fault part is repaired. (Provided that $T_{x}$ is time that the part $i$ correspond)

a) If it is that a fault part is repaired, then renew parts' states: $k=k-1$, at this time if $x_{i}=1$, this part is stored, $y_{i}=y_{i}+1$, otherwise make $x_{i}=1$, this parts immediately begin to work. By judging value of $k$, to decide whether have waiting parts need to be repaired, turn to step 4.2.4.

b) If the redundancy parts break down, then corresponding part, $y_{i}=y_{i}-1, k=k+1$, turn to step 4.2.4.

c) If the work parts break down and see whether have redundancy. If $y_{i}>0$, then switch, $y_{i}=y_{i}-1, k=k+1$, turn to step 4). If $y_{i}=0$, then make $x_{i}=0, k=k+1$, continue to judging whether this part fault causes system fault. If cause system fault, then this simulation failure end, $r=r+1$, turn to step 2), carry on next time simulation; Otherwise turn to step 4.2.4. 


\subsubsection{Count data}

During the period of simulation procedure run, record needed statistic quantities, tidy up and output various statistic quantities after simulation is over.

\section{Applied example}

The system is shown as figure 2, and the table of data is shown as table 1. Parts life and repairing time all obey exponential distributions. Can see from simulative result: probability importance of part 4 is the biggest, this shows parts 4 has important function in the system, this is because part 4 have not storage parts, but because part 2 and part 3 are in parallel connection, and part 2 has already storage parts, so their importance be opposite smaller; Average life of the system is about 70 (hours), the credibility of the system is 0.959 ; Therefore, if want to raise the average life and credibility of system, first from the part 4 commence, by improving credibility or storage parts of part 4 , thus raise the average life and credibility of system.

\section{Conclusions}

It is one of the important steps in simulating that analyze system's states according to fault tree minimal cut-set, can consumedly simplify the complexity of problem. This paper is according to this to simulate and research complicated warm-fund and redundancy repairable system, elaborated to the detailed simulative strategy and calculation of indicators, the last, give one example to shows the feasibility of this method. And that for other similar systems, we still can according to the method and steps elaborated in this paper to carry on simulating and researching, its basic thought is same in essence, just concrete strategy slightly dissimilarity. Moreover, we can also pass modify some parameters of the parts, to observe it to the influence of system reliability under the different condition, then carry on designing and optimizing for the system reliability, give designer provide necessary support of data information.

\section{References}

Billinton R, Li W. (1994). Reliability assessment of electric power systems using Monte Carlo methods. New York: Plenum Press.

Cao Jinhua and Cheng Kan. (1986). Reliability mathematics general introduction. Beijing: Science publishing company. (Chapter 5).

D. M. Malon. (1984). Optimal consecutive-2-out-of-n: F component sequencing. IEEE Trans. Reliab. R-33, 414-418.

D. T. Chiang and S. C. Niu. (1981). Reliability of a consecutive-k-out-of- $n$ : F system. IEEE Trans. Reliab. R-30. 87-89.

Henley EJ and Kumamoto H. (1992). Probabilistic Risk Assessment. IEEE Press.

J. M. Kontoleon. (1980). Reliability determination of a r-successive-out-of- $n$ : F system, IEEE Trans. Reliab. R-29. 437.

Lee WS, Grosh DL, Tillman FA and Lie CH. (1985). Fault tree analysis methods and applications: a review. IEEE Trans Reliab; R-34: 194-302.

Lu J. (1992). Studies on the Monte Carlo simulation methods for estimating network reliability. Master dissertation of the National TsingHua University, ROC

Vesely WE, Goldberg FF, Roberts NH and Haasl DF. (1981). Fault tree handbook. United States Nuclear Regulatory Commission, January.

Xu Shuangwei, Tan Lin and Gong Shiyu. (2007). A reliability simulation approach considering components stand-by redundancy. Electronics product reliability and environment experiment.

Yeh W. (2004). A simple MC-based algorithm for evaluating reliability of a stochastic-flow network with unreliable nodes. Reliab Eng Syst Saf; 83(1): 47-55. 5: (4) 1518. 
Table 1. The table of data (unit: hour)

\begin{tabular}{|c|c|c|c|c|c|c|c|}
\hline parts number & $y_{i}$ & $\lambda_{1}$ & $\lambda_{2}$ & $\mu$ & $W_{N}\left(Z_{i}\right)$ & $M T B F$ & $R(t) \quad(\mathrm{t}=200)$ \\
\hline 1 & 1 & 0.01 & 0.001 & 0.01 & 0.1784 & \multirow{4}{*}{69.79} & \multirow{4}{*}{0.959} \\
\hline 2 & 1 & 0.01 & 0.001 & 0.01 & 0.0843 & & \\
\hline 3 & 0 & 0.01 & - & 0.01 & 0.0397 & & \\
\hline 4 & 0 & 0.01 & - & 0.01 & 0.6976 & & \\
\hline
\end{tabular}

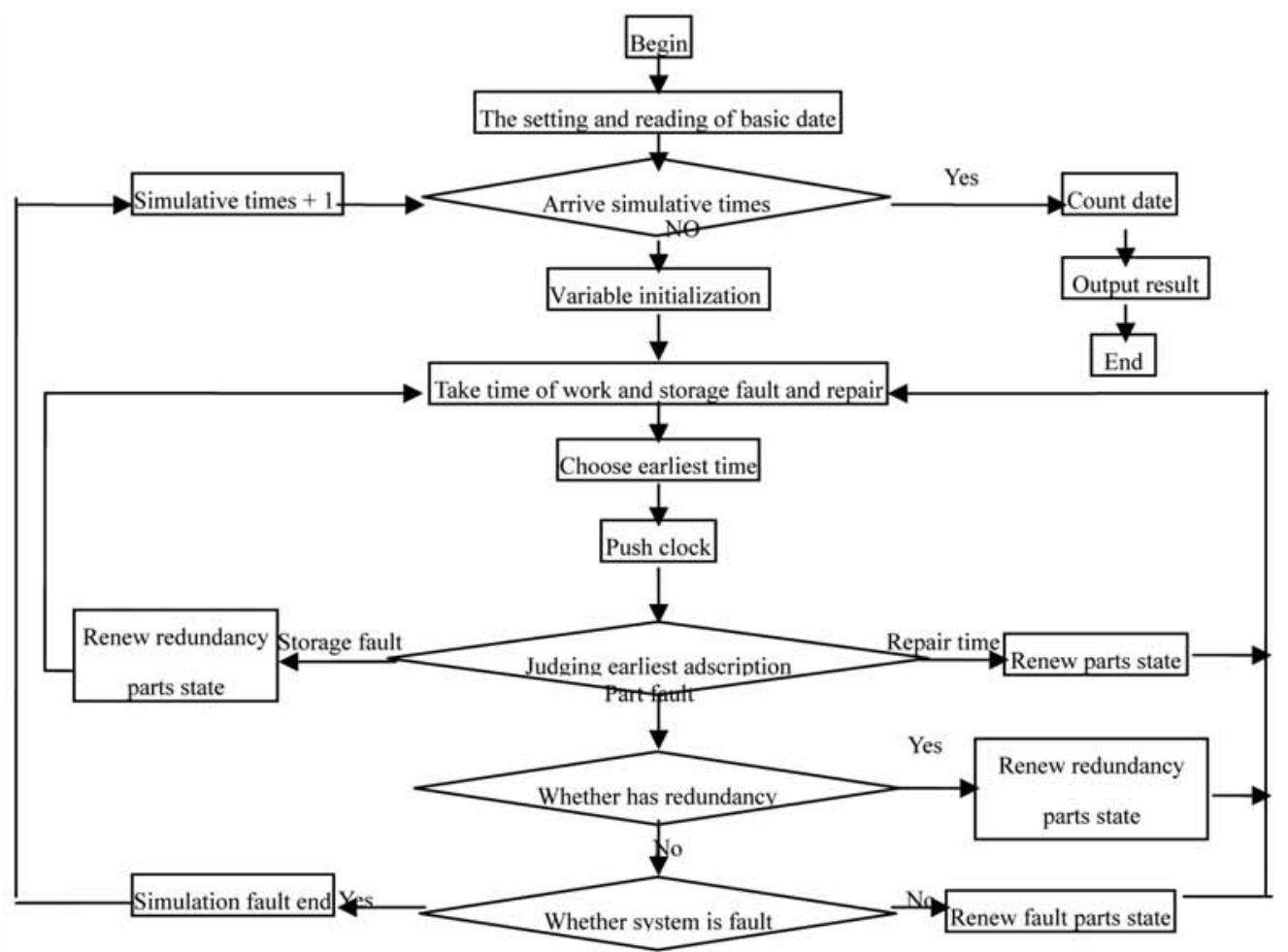

Figure 1. Simulative logic flow chart

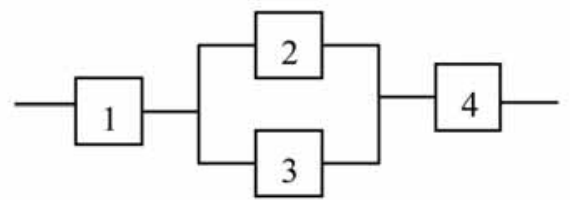

Figure 2. The system of example 